\title{
Hábitos alimentares e de atividade física associados a risco para o desenvolvimento de câncer: prevalência em adolescentes
}

\author{
Dietary and physical activity habits associated with risk for the development of cancer: \\ prevalence in adolescents
}

\section{Wendel Mombaque dos Santos ${ }^{1}$, Renato Xavier Coutinho ${ }^{2}$}

${ }^{1}$ Enfermeiro. Especialista em Enfermagem do Trabalho e Ciências da Saúde. Mestre em Enfermagem, Universidade Federal de Santa Maria, Santa Maria, RS; ${ }^{2}$ Educador Físico. Doutor em Educação em Ciências: Química da Vida e Saúde, Instituto Federal Farroupilha, Santa Maria, RS.

\section{RESUMO}

Objetivos: Avaliar a prevalência de hábitos alimentares e tipo de atividade física associados ao potencial risco para o desenvolvimento de câncer, em uma amostra de adolescentes.

Métodos: Um estudo transversal recrutou estudantes com idade entre 12 e 15 anos, durante o período acadêmico 2011-2012, na cidade de Uruguaiana, estado do Rio Grande do Sul. A coleta de dados foi realizada a partir de um questionário composto por 11 perguntas com variáveis demográficas e comportamentais.

Resultados: A amostra do estudo foi composta por 956 adolescentes de 12 a 15 anos, sendo 498 (52,1\%) do sexo masculino. Entre os 956 adolescentes, $215(22,7 \%)$ praticavam menos de 30 minutos de atividade física por dia, $781(81,7 \%)$ consumiam bebidas açucaradas, 119 $(12,4 \%)$ consumiam mais de 2 drinks por dia de bebida alcoólica, 807 (84,4\%) consumiam carne vermelha, 405 (42,3\%) eram adeptos do chimarrão, $342(35,7 \%)$ consumiam alimentos salgados e $726(75,9 \%)$ consumiam fast-food. As meninas faziam menos atividade física, consumiam menos carne vermelha e mais fast-food do que os meninos. A idade associou-se inversamente ao exercício físico e diretamente ao consumo de bebidas açucaradas e alcoólicas.

Conclusões: A população jovem estudada apresentou comportamentos de alto risco, como baixa atividade física, consumo de alimentos salgados, carnes vermelhas, fast-food, bebidas açucaradas e bebidas alcoólicas, os quais são associados ao aumento na prevalência de vários tipos de câncer.

DESCRITORES: CÂNCER; ADOLESCENTES; NUTRIÇÃO; HÁBITOS ALIMENTARES; FATORES DE RISCO.

\section{ABSTRACT}

Aims: To evaluate the prevalence of dietary habits and of physical exercise with the potential risk for the development of cancer, in a sample of adolescents.

Methods: Cross-sectional study that recruited schoolers aged 12-15 years during the academic year 2011-2012 in the city of Uruguaiana (Brazil). Data collection was performed with a questionnaire containing 11 inquiries with demographic and behavioral variables.

Results: The sample included 956 adolescents, of whom 498 (52.1\%) were boys. Among the 956 participants, 215 (22.7\%) performed less than 30 minutes a day of physical activity; $781(81.7 \%)$ consumed sugary drinks; $119(12.4 \%)$ had at least two alcoholic drinks per day; $807(84.4 \%)$ ate red meat; $342(35.7 \%)$ ate high-sodium snacks; and $726(75.9 \%)$ consumed fast-food. Girls practiced less physical activity, consumed less red meat and more fast-food than boys. Age was inversely associated to physical exercise and positively associated to consuming sugary drinks and alcohol.

Conclusions: The young population we studied presented high-risk behaviors such as low level of physical activity, and consumption of salty foods, red meat, fast food, sugary drinks and alcohol, which are associated with the increase of prevalence of several types of cancer. KEY WORDS: CANCER; ADOLESCENTS; NUTRITION; FOOD HABITS; RISK FACTORS. 


\section{INTRODUÇÃO}

No ano de 2012 houve 14,1 milhões de casos novos e 8,2 milhões de mortes em decorrência do câncer em todo o mundo. No Brasil, para o ano de 2014, estima-se cerca de 400 mil novos casos de câncer, sendo que 12 mil referem-se a cânceres infantis. ${ }^{1} \mathrm{O}$ cenário dos padrões de morbimortalidade em decorrência do câncer pode ser afetado por meio de alimentos, nutrição, atividade física e composição corporal, logo há uma crescente necessidade de conhecimento da prevalência de certos comportamentos, pois a predominância de mortes passou a ser decorrente do estilo de vida associado às patologias. ${ }^{2,3}$

Neste sentido, um aspecto fundamental para a melhoria da qualidade de vida de uma população é a capacidade de compreender os fatores de risco relacionados à alteração de sua saúde. A informação sobre determinado desfecho em saúde deve ajudar a evitar o surgimento deste agravo, assim como influenciar na busca pela prevenção do problema. Portanto, espaços de uso e fluxo populacional, como escolas, universidades, veículos de comunicação e serviços de saúde, são potenciais difusores dessa informação, ainda que a forma de impactar perante a população geral possa ser distinta. ${ }^{4}$

Conforme o manual elaborado pelo Instituto Nacional de Câncer (INCA), os seguintes comportamentos de risco podem levar ao aumento do risco de câncer: consumo de bebidas alcoólicas; consumo de carne vermelha: consumo de chimarrão; consumo de comidas salgadas; consumo de bebidas açucaradas; não ter sido amamentado quando criança; baixa prática de atividade física; consumo de fast-food. ${ }^{3}$

Há escassez de estudos que visem à prevalência de hábitos e comportamentos de risco em adolescentes para o desenvolvimento de câncer. Os hábitos presentes nessa faixa etária tendem a ser mais prejudiciais, visto que quanto maior o tempo de exposição maior será a possibilidade de ação dos fatores de risco. ${ }^{5}$ Dados oficiais sobre a exposição dos adolescentes a comportamentos de risco são imprescindíveis para o monitoramento dos níveis de saúde, auxiliando a implementação e desenvolvimento de políticas e programas educacionais que visem a promoção da saúde. ${ }^{6}$

Assim, devido à grande diversidade de cultura e hábitos nas diferentes regiões do Brasil, surge a necessidade de estudos regionalizados. Sendo a maioria dos estudos originados dos grandes centros, é importante a descentralização das pesquisas, justificadas pelo aumento dos casos de câncer na população brasileira. Deste modo, o presente estudo teve por objetivo avaliar a prevalência de hábitos alimentares com potencial risco para o desenvolvimento de câncer, em adolescentes da região sul do Brasil.

\section{MÉTODOS}

Foi realizado um estudo transversal, em escolas públicas do Município de Uruguaiana/RS, Brasil, no período letivo de 2011 a 2012. Participaram do estudo todas as escolas públicas municipais e estaduais. A população de estudo foi composta por estudantes matriculados em escolas de ensino fundamental, tendo como critério de inclusão a idade entre 12 e 15 anos.

A seleção dos participantes foi randomizada aleatória, tendo por base o cálculo amostral considerando um erro amostral de 3,8\%, com nível de confiança de $98 \%$ para uma população em torno de 20.000 adolescentes, sendo necessária uma amostra de 937 para ser representativa. A coleta de dados foi realizada exclusivamente pelos pesquisadores, através de um questionário elaborado pelos mesmos, contendo questões sobre hábitos alimentares e atividade física referentes ao último mês, assim como características sociodemográficas.

Foram considerados os seguintes fatores de risco, conforme as recomendações do INCA: ${ }^{6}$

- Atividade física - prática inferior a 30 minutos de caminhada diária.

- Bebidas açucaradas - são bebidas com alta densidade energética, com mais de 225-275 kcal/100 g.

- Bebidas alcoólicas - consumo superior a dois drinks por dia ou mais de $30 \mathrm{~g}$ de etanol.

- Carne vermelha - mais de 300 g por semana. É considerada carne vermelha a proveniente de gado bovino, porco e cordeiro, incluindo as carnes processadas.

- Erva mate (chimarrão) - consumo de pelo menos 2 litros por semana, do chá de Ilex paraguariensis, com temperatura igual ou superior $60^{\circ} \mathrm{C}$.

- Alimentos salgados - consumo superior a 5 g por dia. Na prática, avaliado pelas informações sobre os alimentos que eram consumidos diariamente e por meio da estimativa de colheres de chá, quando era adicionado exclusivamente o sal.

- Fast-food - consumo de qualquer quantidade de fast-food. O termo refere-se a alimentos de conveniência disponíveis prontamente, que tendem a ter alta densidade de energia e são consumidos com frequência e em grandes porções.

Para avaliação das variáveis anteriores os alunos listavam os alimentos que consumiam (nome comercial ou genérico) e sua frequência. Após, era realizada classificação conforme as informações nutricionais de cada alimento. 
Previamente à aplicação do questionário, foi realizada a divulgação da pesquisa nas escolas potencialmente elegíveis, explicando como seriam avaliadas as variáveis da pesquisa e solicitando aos participantes assinarem o termo de assentimento e a seus responsáveis o termo de consentimento livre e esclarecido. $\mathrm{O}$ projeto de pesquisa foi aprovado pelo comitê de ética em pesquisa da Universidade Federal do Pampa.

Os dados coletados foram duplamente inseridos no programa Epi Info 7.0 e a análise estatística foi realizada mediante frequência simples e Teste do QuiQuadrado, por meio do software SPSS (Versão 21.0).

\section{RESULTADOS}

A amostra do estudo foi composta por 956 adolescentes de 12 a 15 anos, sendo $498(52,1 \%)$ do sexo masculino, 75 (80\%) com renda familiar superior a um salário mínimo e $380(39,8 \%)$ residentes da periferia da cidade. A Tabela 1 descreve os comportamentos encontrados nos adolescentes entrevistados.

Ao ser avaliada a distribuição dos fatores de risco conforme o sexo, os seguintes fatores apresentaram distribuição significativamente diferente, conforme teste do Qui-quadrado: atividade física, consumo de carne vermelha e consumo de fast-food. Quanto à distribuição dos fatores de risco conforme idade,
Tabela 1. Prevalência de comportamentos de risco para desenvolvimento de câncer em estudantes de escolas públicas da cidade de Uruguaiana, Rio Grande do Sul, no período letivo 2011-2012. Amostra composta por 498 adolescentes do sexo masculino e 458 do sexo feminino.

\begin{tabular}{|c|c|c|}
\hline Variáveis & $\mathrm{n}$ & (\%) \\
\hline \multicolumn{3}{|l|}{ Atividade física } \\
\hline$\leq 30$ minutos/dia & 217 & $(22,7)$ \\
\hline$\geq 30$ minutos/dia & 739 & $(77,3)$ \\
\hline \multicolumn{3}{|l|}{ Consumo de bebidas açucaradas } \\
\hline$\geq 225-275 \mathrm{kcal} / 100 \mathrm{~g}$ & 781 & $(81,7)$ \\
\hline$<225-275 \mathrm{kcal} / 100 \mathrm{~g}$ & 175 & $(18,3)$ \\
\hline \multicolumn{3}{|l|}{ Consumo de bebida alcoólica } \\
\hline$>2$ drinks/dia (10-15 g etanol/drink) & 119 & $(12,4)$ \\
\hline$\leq 2$ drinks/dia (10-15 g etanol/drink) & 837 & $(87,5)$ \\
\hline \multicolumn{3}{|l|}{ Consumo de carne vermelha } \\
\hline$>300 \mathrm{~g} /$ semana & 807 & $(84,4)$ \\
\hline$\leq 300 \mathrm{~g} / \mathrm{semana}$ & 149 & $(15,5)$ \\
\hline \multicolumn{3}{|l|}{ Consumo de chimarrão } \\
\hline$>2$ litros por semana $\left(\mathrm{t}>60^{\circ} \mathrm{C}\right)$ & 405 & $(42,3)$ \\
\hline$<2$ litros por semana $\left(\mathrm{t}<60^{\circ} \mathrm{C}\right)$ & 551 & $(57,6)$ \\
\hline \multicolumn{3}{|l|}{ Consumo de alimentos salgados } \\
\hline$<5 \mathrm{~g} / \mathrm{dia}$ & 342 & $(35,7)$ \\
\hline$>5 \mathrm{~g} / \mathrm{dia}$ & 614 & $(64,2)$ \\
\hline \multicolumn{3}{|l|}{ Consumo de fast-food } \\
\hline Sim & 726 & $(75,9)$ \\
\hline Não & 230 & $(24,0)$ \\
\hline
\end{tabular}

houve diferença significativa com relação à prática de atividade física, consumo de bebidas açucaradas e consumo de bebidas alcoólicas (Tabela 2).

Tabela 2. Fatores de risco para desenvolvimento de câncer, presentes em estudantes de escolas públicas da cidade de Uruguaiana, Rio Grande do Sul, no período letivo 2011-2012, conforme sexo e idade. Amostra composta por 498 adolescentes do sexo masculino e 458 do sexo feminino.

\begin{tabular}{|c|c|c|c|c|c|c|c|c|c|c|c|c|c|c|}
\hline \multirow{3}{*}{ Variáveis } & \multicolumn{5}{|c|}{ Sexo } & \multicolumn{9}{|c|}{ Idade } \\
\hline & \multicolumn{2}{|c|}{ Masculino } & \multicolumn{2}{|c|}{ Feminino } & \multirow{2}{*}{$p$} & \multicolumn{2}{|c|}{12 anos } & \multicolumn{2}{|c|}{13 anos } & \multicolumn{2}{|c|}{14 anos } & \multicolumn{2}{|c|}{15 anos } & \multirow{2}{*}{$p$} \\
\hline & $\mathbf{n}$ & $(\%)$ & n & $(\%)$ & & $\mathbf{n}$ & (\%) & n & $(\%)$ & $\mathbf{n}$ & $(\%)$ & n & $(\%)$ & \\
\hline \multicolumn{15}{|l|}{ Atividade física } \\
\hline$\leq 30$ minutos por dia & 77 & $(8,1)$ & 140 & $(14,6)$ & \multirow{2}{*}{$<0,01$} & 26 & $(2,7)$ & 78 & $(8,2)$ & 67 & $(7,0)$ & 46 & $(4,8)$ & \multirow{2}{*}{$<0,01$} \\
\hline$\geq 30$ minutos por dia & 421 & $(44)$ & 318 & $(33,3)$ & & 161 & $(16,8)$ & 261 & $(27,3)$ & 223 & $(23,3)$ & 94 & $(9,8)$ & \\
\hline \multicolumn{15}{|l|}{ Consumo de bebidas açucaradas } \\
\hline$\geq 225-275 \mathrm{kcal}$ por $100 \mathrm{~g}$ & 406 & $(42,5)$ & 375 & $(39,2)$ & \multirow{2}{*}{0,88} & 161 & $(16,8)$ & 266 & $(27,8)$ & 250 & $(26,2)$ & 104 & $(10,9)$ & \multirow{2}{*}{$<0,01$} \\
\hline$<225-275 \mathrm{kcal}$ por $100 \mathrm{~g}$ & 92 & $(9,6)$ & 83 & $(8,7)$ & & 26 & $(2,7)$ & 73 & $(7,6)$ & 40 & $(4,2)$ & 36 & $(3,8)$ & \\
\hline \multicolumn{15}{|l|}{ Consumo de bebida alcoólica } \\
\hline$>2$ drinks/dia (10-15 g/etanol drink) & 61 & $(6,4)$ & 58 & $(6,1)$ & \multirow{2}{*}{0,84} & 6 & $(0,6)$ & 26 & $(2,7)$ & 49 & $(5,1)$ & 38 & $(4,0)$ & \multirow{2}{*}{$<0,01$} \\
\hline$\leq 2$ drinks/dia (10-15 g/etanol drink) & 437 & $(45,7)$ & 400 & $(41,8)$ & & 181 & $(18,9)$ & 313 & $(32,7)$ & 241 & $(25,2)$ & 102 & $(10,7)$ & \\
\hline \multicolumn{15}{|l|}{ Consumo de carne vermelha } \\
\hline$>300 \mathrm{~g} /$ semana & 401 & $(41,9)$ & 406 & $(42,5)$ & \multirow{2}{*}{$<0,01$} & 161 & $(16,8)$ & 283 & $(29,6)$ & 249 & $(26,0)$ & 114 & $(11,9)$ & \multirow{2}{*}{0,56} \\
\hline$\leq 300 \mathrm{~g} / \mathrm{semana}$ & 97 & $(10,1)$ & 52 & $(5,4)$ & & 26 & $(2,7)$ & 56 & $(5,9)$ & 51 & $(5,3)$ & 26 & $(2,7)$ & \\
\hline \multicolumn{15}{|l|}{ Consumo de chimarrão } \\
\hline$>2$ litros por semana $\left(\mathrm{t}^{*}>60^{\circ} \mathrm{C}\right)$ & 224 & $(23,4)$ & 181 & $(18,9)$ & \multirow{2}{*}{0,08} & 81 & $(8,5)$ & 147 & $(15,4)$ & 117 & $(12,2)$ & 60 & $(6,3)$ & \multirow{2}{*}{0,87} \\
\hline$<2$ litros por semana $\left(\mathrm{t}<60^{\circ} \mathrm{C}\right)^{+}$ & 274 & $(28,7)$ & 277 & $(29)$ & & 106 & $(11,1)$ & 192 & $(20,1)$ & 173 & $(18,1)$ & 80 & $(8,4)$ & \\
\hline \multicolumn{15}{|l|}{ Consumo de alimentos salgados } \\
\hline$<5 \mathrm{~g} /$ dia & 190 & $(19,9)$ & 152 & $(15,9)$ & \multirow{2}{*}{0,11} & 66 & $(6,9)$ & 131 & $(13,7)$ & 107 & $(11,2)$ & 38 & $(4,0)$ & \multirow{2}{*}{0,11} \\
\hline$>5 \mathrm{~g} / \mathrm{dia}$ & 308 & $(32,2)$ & 306 & $(32)$ & & 121 & $(12,7)$ & 208 & $(21,8)$ & 183 & $(19,1)$ & 102 & $(10,7)$ & \\
\hline \multicolumn{15}{|l|}{ Consumo de fast-food } \\
\hline Sim & 347 & $(36,3)$ & 379 & $(39,6)$ & & 137 & $(14,3)$ & 253 & $(26,5)$ & 228 & $(23,8)$ & 108 & $(11,3)$ & \\
\hline Não & 151 & $(15,8)$ & 79 & $(8,3)$ & $<0,01$ & 50 & $(5,2)$ & 86 & $(9,0)$ & 62 & $(6,5)$ & 32 & $(3,3)$ & 0,51 \\
\hline
\end{tabular}

${ }^{*} \mathrm{t}=$ temperatura da água; ${ }^{+}$Menos de 2 litros $\mathrm{OU}$ temperatura abaixo de $60^{\circ} \mathrm{C}$. 


\section{DISCUSSÃO}

Este estudo foi pioneiro em avaliar a prevalência, em adolescentes, de determinados hábitos alimentares e baixa atividade física, reconhecidos como potenciais fatores de risco para o desenvolvimento de câncer, mostrando que a população estudada estava exposta a vários comportamentos de risco.

A ausência de atividade física, sobretudo em meninas e nos escolares entre 13 e 14 anos, aliada com consumo de bebidas açucaradas e consumo de fast-food, verificados neste estudo, levam ao aumento de gordura corporal geral, gordura abdominal e ao maior ganho de peso na idade adulta. A prevalência de pessoas com sobrepeso, independente da idade, vem aumentando nas últimas décadas, tornando-se um problema de Saúde Pública. ${ }^{7,8}$

Nas ultimas décadas as populações latinoamericanas urbanizaram-se e, como consequência, a ingestão de alimentos com alta densidade de energia elevou-se acentuadamente e a atividade física decaiu, com tendência crescente ao sedentarismo. No Brasil cerca de $12 \%$ da população adulta é obesa, considerando como obesidade um índice de massa corporal (IMC) igual ou maior a $30 \mathrm{~kg} / \mathrm{m} 2$. Grupos populacionais com nível socioeconômico baixo são mais afetados, pois possuem poucas opções para uma alimentação saudável e para o desenvolvimento de atividades físicas sob forma de lazer. ${ }^{9}$ Segundo dados da literatura, aumento da gordura corporal elava o risco para o desenvolvimento dos cânceres de esôfago, pâncreas, vesícula biliar, fígado, cólon, reto, mama pós menopausa, endométrio e rins. ${ }^{3}$

O consumo de bebidas alcoólicas constituiu um dos principais fatores de risco presentes entre os adolescentes deste estudo, principalmente na faixa etária entre 14 e 15 anos, sobretudo pelo fácil acesso. Essa utilização pode estar associada às amizades, visto que os amigos exercem grande influência no início e na progressão do consumo de bebidas alcoólicas. ${ }^{8}$ Vários estudos tem demonstrado associação do consumo de álcool com o desenvolvimento de cânceres de cavidade oral, esôfago e laringe, que podem ser provocados pelo resultado da ação direta do álcool na mucosa ou por sua presença na corrente sanguínea e consequente ação sobre os outros sistemas. ${ }^{10-13}$

O consumo de carne vermelha, o qual foi elevado em toda a amostra do estudo, está associado com o aumento do risco para câncer colo-retal em $28 \%$ a $35 \%$, enquanto a carne processada eleva este risco para $20 \%$ a $49 \%{ }^{14,15}$ Embora a associação entre consumo de carne e câncer possa ser parcialmente explicada pelas dietas com alta taxa calórica ou alto teor de gordura, existe um possível papel direto de compostos potencialmente cancerígenos que são encontrados nas carnes, incluindo compostos $\mathrm{N}$-nitrosos, aminas heterocíclicas, ou hidrocarbonetos aromáticos policíclicos. Compostos $\mathrm{N}$-nitrosos são potentes carcinógenos em modelos animais, e incluem as nitrosaminas, que requerem ativação metabólica para serem convertidas numa forma cancerígena, e as nitrosamidas, que não necessitam de ativação. Da mesma forma, aminas heterocíclicas são classificadas como mutagênicas e cancerígenas em animais. Estes e outros compostos presentes em carnes (sais, nitratos, nitritos, ferro heme, gordura saturada, estradiol) têm sido apontados como capazes de aumentar a síntese de DNA e a proliferação celular, aumentar os fatores de crescimento semelhantes à insulina, alterar o metabolismo hormonal, promover o dano dos radicais livres e produzir aminas heterocíclicas carcinogênicas, todos estes, mecanismos que podem promover o desenvolvimento de câncer. ${ }^{16}$

$\mathrm{O}$ consumo de chimarrão (infusão da erva Ilex paraguariensis) aumenta o risco do câncer de esôfago, conforme estudo que foi realizado com uma população de não fumantes e não consumidores de bebida alcoólica. ${ }^{17,18}$ A carcinogenicidade da Ilex paraguariensis seria devida ao seu conteúdo de hidrocarbonetos aromáticos policíclicos, incluindo benzo e pireno, que são classificadas como agentes cancerígenos para seres humanos. ${ }^{19}$ Entretanto, há evidências de que esse vegetal possui propriedades anti-inflamatórias e desempenha um papel na apoptose das células cancerígenas do cólon humano, ${ }^{19}$ o que demonstra que a temperatura da água na preparção do chimarrão pode ter um papel fundamental, e não a erva em si, no desenvolvimento do câncer de esôfago.

A frequente ingestão de altos níveis de sal nos alimentos está associada ao desenvolvimento de câncer de estômago. ${ }^{20,21}$ Conforme aumenta o consumo de sal, o risco para o desenvolvimento deste tipo de câncer cresce progressivamente, sendo que a ingestão de alimentos em conserva com grande quantidade de sal aumenta em $50 \%$ este risco. ${ }^{22,23}$

A estimativa de incidência de câncer na região Sul do Brasil é de 116.300 casos. Os principais tipos de câncer que ocorrem na região Sul, para os quais foram encontrados fatores de risco neste estudo são o de mama (10.370 casos), colón e reto (6.060 casos), estômago (3.490 casos), esôfago (3.020 casos), cavidade oral (2.590 casos) e laringe ( 1.420 casos), os quais representam cerca de $25 \%$ do total dos tipos de câncer na região. ${ }^{1}$ 
A alta prevalência de potenciais fatores de risco para o desenvolvimento de câncer encontrada nos adolescentes deste estudo pode estar associada à elevada prevalência desses tipos de câncer na região sul do Brasil. Os comportamentos de risco iniciados antes da idade adulta podem ser mais danosos, considerando o maior tempo de exposição a que estarão sujeitos esses indivíduos.
Vale ressaltar que este estudo consiste na primeira fase de uma pesquisa longitudinal que irá avaliar em períodos de cinco anos a prevalência e o desenvolvimento de câncer nesta coorte, de forma a ser verificada a real incidência dessa doença na presença de exposição a hábitos de risco.

\section{REFERÊNCIAS}

1. Brasil. Estimativa 2014: Incidência de Câncer no Brasil. In: Vigilância CdPe. Rio de Janeiro: INCA; 2014.

2. Ramis TR, Mielke GI, Habeyche EC, Oliz MM, Azevedo MR, Hallal PC. Tabagismo e consumo de álcool em estudantes universitários: prevalência e fatores associados. Revista Brasileira de Epidemiologia. 2012;15:376-85.

3. Brasil. Alimentos, nutrição, atividade física e prevenção de câncer: uma perspectiva global. INCA. 2007.

4. Borges TT, Rombaldi AJ, Knuth AG, Hallal PC. Conhecimento sobre fatores de risco para doenças crônicas: estudo de base populacional. Cadernos de Saúde Pública. 2009;25:1511-1520.

5. Presti PdF, Macedo CRD, Caran EM, Rodrigues AHD, Petrilli AS. Estudo epidemiológico de câncer na adolescência em centro de referência. Revista Paulista de Pediatria. 2012;30:210-6.

6. Farias Júnior JC, Nahas MV, Barros MVG, et al. Comportamentos de risco à saúde em adolescentes no Sul do Brasil: prevalência e fatores associados. Revista Panamericana de Salud Pública. 2009;25:344-52.

7. Terres NG, Pinheiro RT, Horta BL, Pinheiro KAT, Horta LL. Prevalência e fatores associados ao sobrepeso e à obesidade em adolescentes Revista de Saúde Pública. 2006;40:627-33.

8. Coutinho RX, Santos WM, Folmer V, Puntel RL. Prevalência de comportamentos de risco em adolescentes. Cadernos Saúde Coletiva 2013;21:441-9.

9. Wünsch Filho V, Antunes JLF, Boing AF, Lorenzi RL. Perspectivas da investigação sobre determinantes sociais em câncer. Physis: Revista de Saúde Coletiva. 2008;18:427-50.

10. Wünsch V. The epidemiology of laryngeal cancer in Brazil. Sao Paulo Medical Journal. 2004;122:188-94.

11. Sartor SG, Eluf-Neto J, Travier N, et al. Riscos ocupacionais para o câncer de laringe: um estudo caso-controle. Cadernos de Saúde Pública. 2007;23:1473-81.

12. Scheidt JHG, Yurgel LS, Cherubini K, Figueiredo MAZd, Salum FG. Characteristics of oral squamous cell carcinoma in users or non users of tobacco and alcohol. Revista Odonto Ciência. 2012;27:69-73.

13. Carrard VC, Pires AS, Paiva RL, Chaves ACM, Filho MSA. Álcool e Câncer Bucal: Considerações sobre os Mecanismos Relacionados. Revista Brasileira de Cancerologia. 2008;54(1):7.

14. Zandonai AP, Sonobe HM, Sawada NO. Os fatores de riscos alimentares para câncer colorretal relacionado ao consumo de carnes. Revista da Escola de Enfermagem da USP. 2012;46:234-9.

15. GuimarãesRM, Rocha PGM, Muzi CD, Ramos RS. Increase income and mortality of colorrectal cancer in Brazil, 2001-2009. Arquivos de Gastroenterologia. 2013;50:64-9.

16. Genkinger JM, Koushik A. Meat consumption and cancer risk. PLoS Med. 2007;4(12):e345.

17. Ronco AL, Barrios E, Fierro L, Balbi J, Larrinaga MT, De Stéfani E. Risk factors for esophageal cancer in non-smokers and non-drinkers: a case-control study in Uruguay. Revista Brasileira de Epidemiologia. 2004;7:383-91.

18. Dietz J, Pardo SH, Furtado CD, Harzheim E, Furtado AD. Fatores de risco relacionados ao câncer de esôfago no Rio Grande do Sul. Revista da Associação Médica Brasileira. 1998;44:269-72.

19. Andrici J, Eslick GD. Mate consumption and the risk of esophageal squamous cell carcinoma: a meta-analysis. Diseases of the esophagus: official journal of the International Society for Diseases of the Esophagus/I.S.D.E. 2013;26(8):807-16.

20. Magalhães LP, Oshima CTF, Souza LG, Lima JMd, Carvalho Ld, Forones NM. Variação de peso, grau de escolaridade, saneamento básico, etilismo, tabagismo e hábito alimentar pregresso em pacientes com cancêr de estômago. Arquivos de Gastroenterologia. 2008;45:111-16.

21. Gómez Zuleta M, Otero Regino W, Ruiz Lobo X. Factores de riesgo para cáncer gástrico en pacientes colombianos. Revista Colombiana de Gastroenterologia. 2009;24:134-43.

22. Gonzalez CA, Sala N, Rokkas T. Gastric cancer: epidemiologic aspects. Helicobacter. 2013;18 Suppl 1:34-8.

23. Delahaye F. Should we eat less salt? Archives of cardiovascular diseases. 2013;106(5):324-32. 